Artículo original

\title{
INFLUENCIA DEL NIVEL DE PRECIOS Y LA VALORACIÓN DE BENEFICIOS SOBRE EL CONSUMO DE PRODUCTOS ORGÁNICOS EN TACNA-PERÚ
}

\author{
INFLUENCE OF PRICE LEVEL AND VALUATION OF BENEFITS \\ ON CONSUMPTION OF ORGANIC PRODUCTS IN TACNA-PERU
}

AleXANDRA DUTSI CARRASCO RIVERA ${ }^{1}$

(D) https://orcid.org/0000-0001-9608-6029

NELly EsPinoza OSNAYO ${ }^{2}$

(D) https://orcid.org/0000-0002-3275-8604

Raúl Cartagena Cutipa ${ }^{3}$

(D) https://orcid.org/0000-0002-3419-7097

Información del artículo: Recibido: 21/01/2021 Aceptado: 01/06/2021 Publicado: 28/06/2021

${ }^{1,2}$ Escuela de Ingeniería Agroindustrial, Universidad Privada de Tacna ${ }^{3}$ Docente en la Escuela de Ingeniería Agroindustrial, Universidad Privada de Tacna E-mail: ${ }^{1}$ bcarrasco@hotmail.com, ${ }^{2}$ nellyespinozaosnayo@gmail.com, ${ }^{3}$ raucartagenac@upt.pe 


\section{Resumen}

Para establecer la relación del nivel de precios, la valoración de beneficios y factores asociados sobre el consumo de productos orgánicos en la ciudad de Tacna, se tuvo que medir los bajos niveles de consumo; el nivel de conocimiento, el nivel de precios, la valoración de beneficios, y el probable efecto de la pandemia COVID 19. La investigación fue de carácter descriptivo y de corte trasversal. La técnica utilizada fue la encuesta, diseñando un cuestionario con 23 preguntas que fueron validadas por alfa de Cronbach de 0,872 , el instrumento se aplicó virtualmente a 382 personas entre 18 y 64 años de edad del distrito de Tacna. Para los análisis la muestra fue segmentada en consumidores y no consumidores. Los resultados arrojaron que no existen relaciones significativas $($ sig. $>0,05)$ entre el nivel de consumo con; nivel de conocimiento, la valoración de beneficios, ni los efectos producidos a causa de la pandemia COVID 19. No obstante, sí se encontró una relación significativa aunque débil (Coeficiente de Spearman 0,011) entre los niveles de consumo y el nivel de precios con un nivel de significancia del $95 \%$, demostrándose que el bajo consumo de productos orgánicos está relacionado a los costos altos que tienen en comparación a los productos convencionales, no importando los demás factores a la hora de comprarlos. Respecto a los no consumidores se encontró relaciones altamente significativas (sig. $<0,000$ ) entre las razones de no consumo con; el nivel de precios, el nivel de estudios y desconocimiento. Finalmente se concluye en la ciudad de Tacna, los bajos niveles de consumo se deben principalmente al precio, y otros factores como precios altos y desconocimiento

Palabras claves: Productos orgánicos; nivel de consumo; valoración de beneficios.

\section{Abstract}

In order to establish the relationship between the level of prices, the valuation of benefits and associated factors on the consumption of organic products in the city of Tacna, it was necessary to measure the low levels of consumption, the level of knowledge, the level of prices, the valuation of benefits, and the probable effect of the COVID 19 pandemic. The technique used was the survey, designing a questionnaire with 23 questions that were validated by Cronbach's alpha of 0.872 , the instrument was applied virtually to 382 people between 18 and 64 years of age in the district of Tacna. For the analyses, the sample was segmented into consumers and non-consumers. The results showed that there were no significant relationships (sig. $>0.05$ ) between the level of consumption and the level of knowledge, the valuation of benefits, or the effects produced by the COVID 19 pandemic. However, a significant but weak relationship (Spearman's coefficient 0.011) was found between consumption levels and price levels with a significance level of $95 \%$, showing that the low consumption of organic products is related to the high costs they have in comparison to conventional products, other factors being irrelevant when purchasing them. Regarding non-consumers, highly significant relationships were found (sig. < 0.000) between the reasons for non-consumption and price level, level of education and lack of knowledge. Finally, it is concluded that in the city of Tacna, the low levels of consumption are mainly due to price, and other factors such as high prices and lack of knowledge.

Keywords: Organic products; level of consumption; valuation of benefits. 


\section{Introducción}

El consumo de alimentos inocuos y con beneficios adicionales a los comunes están siendo en la actualidad los más demandados, principalmente por los sucesos inesperados que en la actualidad vive la humanidad debido al COVID, existen distintos tipos de productos que el consumidor demanda según su interés, de conservar la salud, estilos de vida saludable, compromisos con el medio ambiente, y otros beneficios. En ese sentido los productos orgánicos son a aquellos alimentos que se consiguen sin la intervención de fertilizantes, herbicidas o pesticidas químicos en todas las etapas de su producción y en los suelos donde son cultivados (Cisneros 2012), otras definiciones más extensas indican que son productos vegetales o animales o sus derivados que se elaboran con sustancias naturales, es decir no se emplean productos químicos, tales como plaguicidas, herbicidas, insecticidas, entre otras sustancias tóxicas o aditivos en ninguna etapa del proceso de producción ni en la materia prima que se usa hasta llegar al producto final (Posada, 2018 ).

Y con el interés de los beneficios que provee, en la actualidad la demanda de productos orgánicos ha incrementado en países desarrollados. Actualmente el mercado de productos orgánicos en el Perú es bajo y la mayor parte de la producción se vende al exterior, el Perú exporta principalmente banano, café, nueces, cereales especias entre otros. No obstante en el mercado interno, es muy escaso el consumo debido a la disponibilidad y la oferta, además de otros factores como la creencia que su consumo solo es en niveles socioeconómicos altos tal como afirma (Jurado 2009), pero a diferencia de años anteriores existen mayores iniciativas de comercialización a nivel interno, en el caso local, según Soto (2015) en los últimos años la demanda de alimentos creció en un $70 \%$, asociado a ello, la oferta de alimentos orgánicos como hortalizas, frutas, tubérculos y plantas medicinales también aumentaron, pero aun parecen ser insuficientes, porque se identifica que menos de $3 \%$ compran o los han comprado, destacándose que no son consumidores habituales, bajo estas consideraciones, además de lo ya explicado; tales como el desconocimiento de sus propiedades, los beneficios que aportan, sus sobrecostos y oferta disponible y los canales de comercialización parecen ocasionar la escasa demanda y oferta a nivel local. Así mismo en el último año, debido a la pandemia ocasionada por el COVID 19 parecen haber influido en la oferta y consumo del mismo.

Por ello, es importante conocer los niveles de consumo, además si este factor está relacionado con el conocimiento de sus propiedades, los niveles de precios, la valoración de beneficios y cuáles son los efectos que podrían haber ocasionado la ocurrencia de la pandemia. La información aportada puede ser de utilidad para los consumidores y productores; los primeros a distinguir mejor y perfilar su consumo y los segundos a aprovechar las tendencias de consumo para replantear su producción de acuerdo a la demanda de los consumidores. Ante esta realidad surgen diversas tendencias de consumo, tales como los que optan por el estilo vegano del cual su alimentación es principalmente el consumo de vegetales y alimentos sin proceso, otros que optan por consumir alimentos mínimamente procesados en su dieta, otros que optan por consumir alimentos cultivados sin el uso de agroquímicos o los denominados orgánicos.

El consumo de alimentos orgánicos ha cobrado especial importancia en los últimos años, ello ha obligado a la implementación de sistemas de certificación, garantizan a los consumidores que no se han utilizado agroquímicos sintéticos ni materiales transgénicos durante los procesos de producción y en el caso de los productos de origen animal, que se han respetado aspectos relacionados con el bienestar de los mismo, respecto a su comercialización, se conoce que el $85 \%$ de mercados de países europeos comercializan productos orgánicos (kramer, 1998) y que en los próximos 30 años la demanda de alimentos en el mundo crecerá en $30 \%$, principalmente en productos orgánicos.Esta situación es particular a nivel local, si bien es cierto se puede encontrar productos orgánicos en los mercados de Tacna, el consumidor parece no tener claro de los beneficios de este tipo de alimentos.

Por otro lado, es importante conocer cuál es la valoración de los beneficios que otorgan los consumidores a los productos orgánicos, principalmente a la salud, así mismo como valoran los productos al comprar los productos, con la finalidad de valorar la producción orgánica. También es 
importante por su contribución al conocer si el comportamiento del consumidor de productos orgánicos está relacionado al conocimiento, su preocupación por su salud, la preocupación por el medio ambiente, siendo factores que pueden ayudar a entender las características de alimentos orgánicos que busca el consumidor. El trabajo es relevante además porque permite establecer las tendencias actuales de consumo del consumidor, pero principalmente sobre los no consumidores, quienes pueden ser un nicho importante y mercado cautivo que al conocer sus expectativas se puede buscar satisfacerlas. Por ello es importante conocer cuál es la necesidad de consumo y qué tipo de productos orgánicos demanda, que tipo de valoración da a sus propiedades y cuál es la disponibilidad a pagar por los mismos.

Narro y Yánac (2018) acerca de la demanda de productos orgánicos, indica que el nivel de conocimiento y la valoración de beneficios de los alimentos orgánicos de un consumo elevado, teniéndose en consideración que la valoración de beneficios tenía una mayor repercusión, reincidiendo en los consumidores con alto grado de concientización al cuidado de la salud. Salgado y Beltrán (2011) demuestra que la conducta del consumidor, y los aspectos demográficos desempeñan un resultado relevante en el consumo sostenible. Andrade y Ayaviri (2018) manifiesta que entre los primordiales obstáculos para el crecimiento del consumo de productos orgánicos tenemos, baja oferta disponible, falta de garantía de orgánicos, vida útil corta y precios elevados; entre los productos orgánicos principalmente consumidos son verduras, hortalizas y frutas orgánicas; se determinó que el nivel de ingresos de los consumidores y la escolaridad de la población no repercuten sobre la demanda y consumo de productos orgánicos.

Higuchi (2015) comprobó que personas con mayor estatus socioeconómicos y educación tienen mayor disposición al momento de adquirir los alimentos orgánicos; Diaz et al., (2015) expresa que, indiscutiblemente la tendencia de consumo presentó un $44 \%$ y que el género femenino tiene una mayor incidencia al momento de la adquisición de los alimentos, y en efecto las personas encuestadas tienen un mayor grado Nivel Socio Económico (NSE) y estudios superiores; que las características socioeconómicas que influyen en el consumo son: elevados precios, desconocimiento de beneficios de los alimentos orgánicos y la falta disponibilidad de alimentos en el mercado, los productos convencionales tienen a tener mayor inclinación por ser considerados productos frescos y saludable, el consumidor los reconoce por la presentación y el sello de certificación. Lacaze (2009) manifiesta que los que consumen alimentos orgánicos tienen mayor disponibilidad a pagar precios elevados a diferencia de los que consumen alimentos convencionales, de donde se infiere que los alimentos que tengan mayor presentación, beneficios y calidad en el producto su consumo serán de mayor preferencia; en estadística se procedió a realizar la regresión logísticas para analizar los datos binomiales para verduras de hoja, harina de trigo integral, pollo fresco y aromático. Así mismo los consumidores tienen mayor disposición al momento de adquirir los alimentos orgánicos cuando tienen mayor información sobre un correcto envasado y etiquetado; además En el caso particular de los alimentos frescos, los consumidores mencionan lo peligroso que pueden ser para la salud consumir alimentos con sustancias nocivas en alimentos convencionales, como químicos y pesticidas en las verduras y las hormonas que inyectan al pollo es por ello que prefieren consumir alimentos orgánicos.

Para Evans y Tveteras (2011), Perú, tiene una superioridad para posicionarse como uno de los principales países para la producción de alimentos orgánicos gracias a sus características y recursos naturales estratégicos como país marítimo, amazónico, andino y con proyección bioceánica (Proexpansión 2014). En agosto del 2001 Perú brindo oportunidades a agricultores (RAAA 2008); lo cual obtuvo un elevado crecimiento en la agricultura orgánica, (especialmente los principales productos certificados se encuentran el café, plátanos, cacao, quinua y nueces o castañas de Brasil (FIBL e Ifoam 2014). A demás en el año 2012, el Perú se ubicó entre los 10 países con la mayor cuantía de alimentos orgánicos y era el quinto en América Latina en extensión de tierras agrícolas destinadas a productos orgánicos, contando con un territorio general de cultivación alrededor de 305.000 hectáreas en todo el estado, lo que implica a más de 47.000 productores (FIBL e Ifoam 2014). Paul y Rana (2012) evaluó la causa de los factores estadísticos, de donde se infiere que el comprador con mayor cultura tiende a consumir más alimentos biológicos, calificando la influencia del consumidor al 
momento de la adquisición de los alimentos, comprobando que los entrevistados con mayor educación y cultura su consumo es mayor en alimentos orgánicos. Dando como resultado los principales motivos para comprar alimentos biológicos, se comprobó que por raciocinio de salubridad (42\%), empaque ecológico (5\%) y tecnología no contaminante (2\%). Demostró que los encuestados adquieren productos orgánicos por treinta motivos de salud. Quiere decir que existe una asociación de beneficios por la salud y el impulso por la compra de alimentos biológicos; a su vez la falta disponibilidad de alimentos en centros de abasto es uno de los principales motivos de los consumidores para la no compra, seguida del costo del producto, características organolépticas del alimento y publicidad.

Saucedo (2010) dio a conocer que Perú está orientado a la competitividad y estrategia de planteamiento, así mismo a nivel nacional producen y exportan alimentos orgánicos, especialmente el café y cacao representando una demanda de $3 \%$ y $97 \%$. Reyes et al., (2014) dentro de los factores asociados al plan de marketing señala al crecimiento exponencial a nivel mundial y una mayor predisposición de la población al consumo de productos orgánicos, que estos productos se hallen libres de químicos y pesticidas, y el pedido a las empresas de acrecentar una cultura responsable socialmente. Dentro de los objetivos están el posicionar a EcoMarket, como uno de los primeros minimarket dedicados a productos orgánicos debidamente certificados en nuestro país y principal difusor de actividades aliada a una propuesta de vida saludable y ecológica, así mismo lograr el 2,2\% de contribución del mercado en el primer año, representado esto a un total de $S / 5,998,320$ de ventas anuales. Sabiendo esto se asociará a valores como: salud, ecologismo, cultura y ética. De igual modo Cardozo et al., (2019) sobre la comercialización, los consumidores buscan mejorar sus hábitos alimenticios saludables buscando encontrar un producto y servicio innovador. Existiendo ya en el mercado diversas opciones de alimentos saludables como restaurantes o delivery que brindan productos como ensaladas, comida baja en grasas, etc. pero ninguna cuenta con un plan de comidas en base a insumos orgánicos complementado con un asesoramiento nutricional. Al contrario Ortega y Gabino (2013) acerca de la adopción de tecnologías orgánicas por productores concluye que a mayor disponibilidad de insumos orgánicos, participación en organizaciones e información de tecnologías orgánicas existe una alta posibilidad de adquirir tecnologías orgánicas; y a menor tamaño del cultivo alta posibilidad de adquirir tecnologías orgánicas. Por el contrario, los factores como conocimiento sobre tecnologías orgánicas, mano de obra, expectativa de ingresos, acceso al crédito, edad del productor y grado de instrucción no influyen en la adquisición de tecnologías orgánicas en la Yarada.

Bermejo (2018) Realizó una investigación sobre los factores que influyen en la intención de compra de alimentos orgánicos, en consumidores del distrito Coronel Gregorio Albarracín Lanchipa en el año 2018. Para esto se realizó una encuesta a 385 consumidores. El análisis estadístico tiene una seguridad de $99 \%$, donde demuestra que, si la edad del consumidor aumenta en un año en promedio, la probabilidad de intención de compra de alimentos orgánicos se incrementa en $0,7 \%$. De ser el consumidor hombre en promedio, la intención de compra disminuye en $15,9 \%$. Por otro lado, si el consumidor realiza rutinas de ejercicio regularmente en promedio, la intención de compra se eleva en $23,2 \%$.

\section{Objetivo}

Determinar la relación del nivel de precios y la valoración de beneficios sobre el consumo de productos orgánicos en la ciudad de Tacna.

\section{Metodología}

La investigación es de tipo no experimental y transversal, porque es un proceso sistemático de recojo de información en el que no se manipula las variables. Es un estudio transversal porque se realizará en un periodo de tiempo determinado. Considerando que la investigación es de tipo 
descriptiva, se empleó como principal técnica; la encuesta, que implica la formulación de un cuestionario para aplicar a la muestra de estudio, por tanto, las actividades para su ejecución se describen en las siguientes acciones.

- Formulación de los indicadores de las variables que se describen en la matriz de consistencia

- Elaboración de escalas de medición de preguntas de cuestionario sobre la base de indicadores

- Formulación de procedimientos codificación de respuestas.

- Digitalización de cuestionarios en medios virtuales

La validación de instrumentos es importante, por ello para la validación del instrumento se realizó un análisis de concordancia interna de los contenidos de las preguntas y su forma de aplicación que estandarice los procesos de recolección de la información y su posterior análisis. Validación del cuestionario mediante la aplicación de una prueba piloto y determinación del porcentaje de fiabilidad. La recolección de la información consistió en aplicar el cuestionario de forma virtual de acuerdo al siguiente procedimiento: La aplicación de la encuesta en forma digital, fue ejecutado a través de la herramienta Google Forms de libre uso, se identificó como unidad de análisis es población del distrito de Tacna, luego del recojo de información se realizó la categorización en función de las variables y se formuló las variables en el software estadístico IBM SPSS V. 25 y posteriormente se codificó las respuestas de cada encuestado, con la finalidad de generar una data que sea accesible para hacer los análisis. Para efectuar la investigación se empleó un cuestionario que consta de 23 preguntas de respuestas cerradas estructuradas con categorías o escalas, dicotómicas politomicas y de Likert. La investigación gira en torno a la variable Influencia del nivel de precios, Valoración de beneficios al consumir productos orgánicos y consumo de productos, para lo cual se consideró los trabajos Narro y Yánac (2018), se estableció las dimensiones de las variables con sus indicadores y niveles. Niveles de consumo de alimentos orgánicos (Frecuencia de consumo, Argumentos de elección en la compra, Cantidad de alimentos orgánicos adquirida y Grupo de alimentos adquiridos)

Niveles de Conocimiento (Conocimiento de las propiedades y Conocimientos generales)

Nivel de precios (Argumentos de elección en la compra de alimentos orgánicos y Factores que ejercen mayor influencia sobre la compra de alimentos orgánicos)

Valoración de beneficios (Identificación de los alimentos orgánicos, Beneficios Esperados y Motivos de consumo)

Consumo de productos orgánicos en tiempo de COVID 19 (Aumento o disminución del consumo, Incremento de precios, Variación de la disponibilidad y Dificultades para adquirir)

La muestra de estudio fue de carácter probabilístico, con el objetivo de ser representativa de la población, según la información obtenida de INEI (2017), la población determinada según "La encuesta de Población y Viviendas efectuada el año 2017", para calcular las muestras se selecciona la población del distrito de Tacna entre 18 y 64 años de edad que son 56977 pobladores. El cálculo de la muestra se emplea la fórmula reportada por Hernández, Fernández y Baptista (2010). Por lo tanto, la muestra de estudio calculada fue 382 personas en total. Para la presentación de la información se emplea estadística descriptiva y luego se calculan los modelos teóricos objetivo de identificar el modelo teórico más adecuado para representar la población de la cual proceden los datos y luego representarlos en gráficos y estadísticos que permiten explorar a priori.

\section{Resultados}

Los resultados son presentados por consumidores y no consumidores, que se reflejan con información demográfica, conocimiento, preferencias de consumo, valoración de beneficios y nivel de precios. En la primera parte se analiza a los consumidores que son una muestra de 175 y los no consumidores de 207.

Los datos sociodemográficos que se midieron en el estudio fueron; genero, edad, nivel de estudios, ingreso familiar y ocupación. Al analizar el género de los encuestados se tiene en cuenta que 
el género tiene puede influir en la decisión de compra de productos del hogar, tales como los productos de consumo inmediato como los productos orgánicos, diversos estudios (Ocampo, 2004; Lipovetsky y Naranjo, 1999) demuestran que en los hogares los hombres son responsables de las decisiones en un área, mientras que las mujeres lo hacen en otras tales como hacer el mercado para la semana (Gentile y Rodriguez, 2002). La muestra de estudio estuvo compuesta por $75,43 \%$ de población de género femenino, mientras que el $24,57 \%$ fue del género masculino. Al ser una muestra aleatoria, se observa que no existe la proporcionalidad entre la cantidad de población según género, no obstante, el estudio está relacionado más al género femenino, lo que pudo haber primado sobre las proporciones de género que diligenciaron las encuestas. La decisión de consumo y compra de un producto orgánico podría estar asociado a la edad del consumidor, por ello es importante analizar la edad de los encuestados, en el estudio se refleja que el mayor porcentaje estuvo compuesto por personas entre 20 a 35 años con un $48 \%$, seguido de personas entre 35 a 45 años con una frecuencia de $22,29 \%$.

Tabla 1

Características sociodemográficas de los encuestados

\begin{tabular}{|c|c|c|}
\hline & $\mathbf{n}$ & $(\%)$ \\
\hline \multicolumn{3}{|c|}{ Género } \\
\hline Hombre & 43 & 24,6 \\
\hline Mujer & 132 & 75,4 \\
\hline Total & 175 & 100,0 \\
\hline \multicolumn{3}{|c|}{ Edad } \\
\hline Menor a 20 & 5 & 2,9 \\
\hline $20-35$ & 84 & 48,0 \\
\hline $35-45$ & 39 & 22,3 \\
\hline $46-55$ & 35 & 20,0 \\
\hline $56-65$ & 11 & 6,3 \\
\hline Mayor a 65 & 1 & 0,6 \\
\hline Total & 175 & 100,0 \\
\hline \multicolumn{3}{|c|}{ Nivel de ingresos familiar } \\
\hline menos de S/ 1300,00 & 37 & 21,1 \\
\hline Entre S/ 1300,00 y S/ 2 480,00 & 60 & 34,3 \\
\hline Entre S/ 2 481,00 y S/ 3 970,00 & 42 & 24,0 \\
\hline Entre S/ 3971,00 y S/ 7 020,00 & 27 & 15,4 \\
\hline Entre S/ 7 021,00 y S/ 12 660,00 & 7 & 4,0 \\
\hline Más de S/ 12 660,00 & 2 & 1,1 \\
\hline Total & 175 & 100,0 \\
\hline \multicolumn{3}{|c|}{ Nivel de estudios } \\
\hline Secundaria & 6 & 3,4 \\
\hline Técnico & 30 & 17,1 \\
\hline Universitario & 116 & 66,3 \\
\hline Maestría/Doctorado & 23 & 13,1 \\
\hline Total & 175 & 100,0 \\
\hline \multicolumn{3}{|c|}{ Ocupación } \\
\hline Empleado & 75 & 42,9 \\
\hline Independiente & 63 & 36,0 \\
\hline Pensionado & 3 & 1,7 \\
\hline Empresario & 6 & 3,4 \\
\hline Ama de casa & 12 & 6,9 \\
\hline Otros & 16 & 9,1 \\
\hline Total & 175 & 100,0 \\
\hline
\end{tabular}


La ocupación laboral comprende la función laboral que tiene la población encuestada y delimita su competencia generalmente se utiliza la denominación, se parecía que la muestra de estudio está representada en mayor cantidad por empleados con 42,86\% seguida de independientes con 36 $\%$. De forma similar a los indicadores sociodemográficos anteriores, el nivel de formación podria influir en el consumo de productos organicos, se aprecia que la mayor parte de población respecto al nivel de estudios tienen nivel de formación universitaria con el 66, $29 \%$, asi mismo no participan del estudio población con menor nivel de estudio.

\section{Figura 1}

Productos orgánicos consumidos (\%)

Hortalizas y frutas frescas

Granos e infusiones (te, café,.

$$
\text { ... }
$$

Legumbres

Hierbas y condimentos

$$
\text { Lácteos }
$$

Carnes (rojas y/o blancas)

Panadería y galletería

Otro tipo

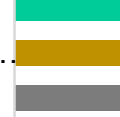

$$
13.60 \%
$$

$11.10 \%$

$8.30 \%$

$8.30 \%$

$6.30 \%$

$5.80 \%$

$4.80 \%$

$2.30 \%$

Figura 2

Productos orgánicos más consumidos

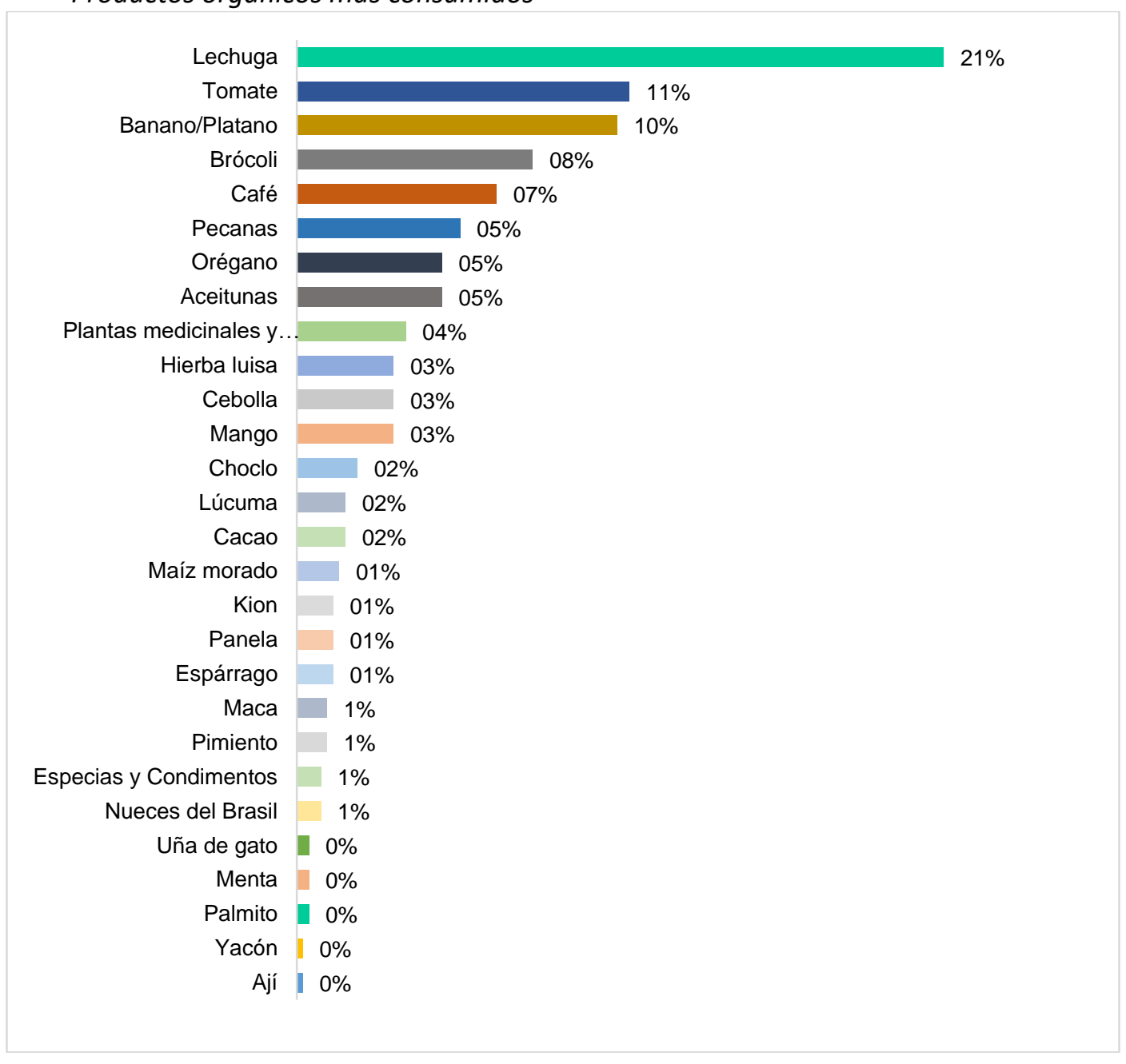


Respecto al tipo de productos específicos que consumen los encuestados, se les consultó que especifiquen al menos tres productos que consumían con frecuencia, donde se evidencia los productos orgánicos producidos en el Perú, donde el objetivo es destacar los tres productos más consumidos, resume tres productos más consumidos: Lechuga con $21 \%$; tomate con $11 \%$ y plátano con $10 \%$.

el lugar donde consumidor de productos orgánicos compra, en primer lugar se observa que las compras las realiza en el mercado local (42\%), mientras que un $17 \%$ lo hace en supermercados, seguida de tiendas especializadas. Al respecto en un estudio realizado por Andrade y Ortiz (2008), encontraron que el lugar preferido de compra de la población ecuatoriana es principalmente los supermercados $(69,3 \%)$, seguido de mercados o ferias libres con $26,7 \%$, mientras que en países desarrollados como Estados Unidos y Canadá, la compra es principalmente en supermercados (49\%) y tiendas especializadas (48\%) según Lutterbeck (2001). Estas diferencias se deberían a los estados de segmentación de dichos productos, así como la disponibilidad y variedad.

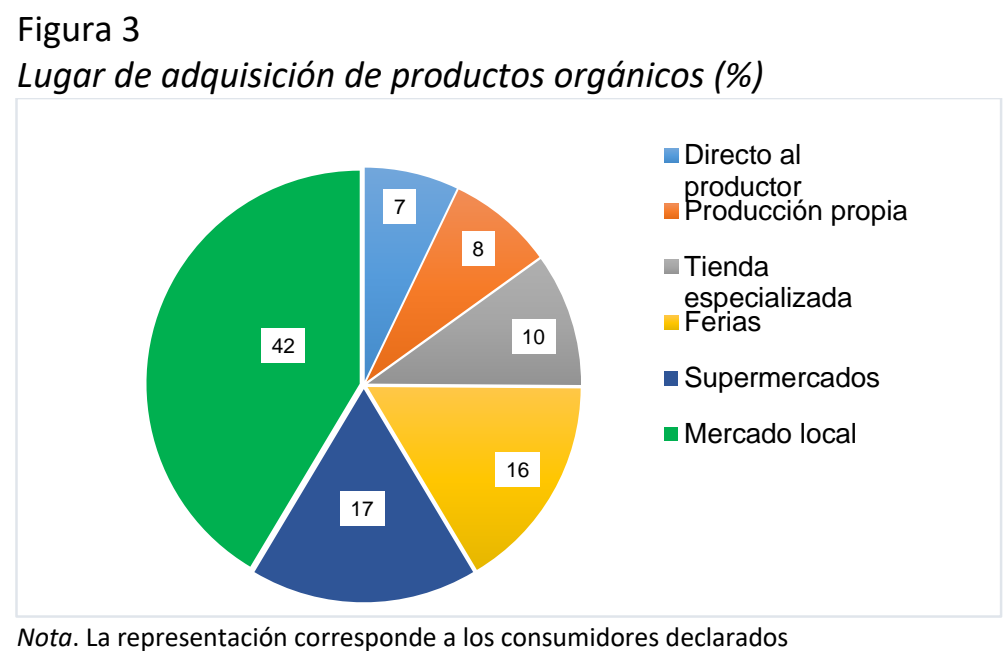

Con la finalidad de analizar de forma general el conocimiento que posee el consumidor de productos orgánicos, se agrupó las variables de conocimiento evaluadas por grupos de productos, donde se observa que en general los consumidores no tendrían conocimiento adecuado de las propiedades de los productos orgánicos, tal como se evidencia la representación de la categoría "bajo conocimiento" con el $77,14 \%$.

Figura 4

Representación de los niveles de conocimiento general de las propiedades de los productos orgánicos

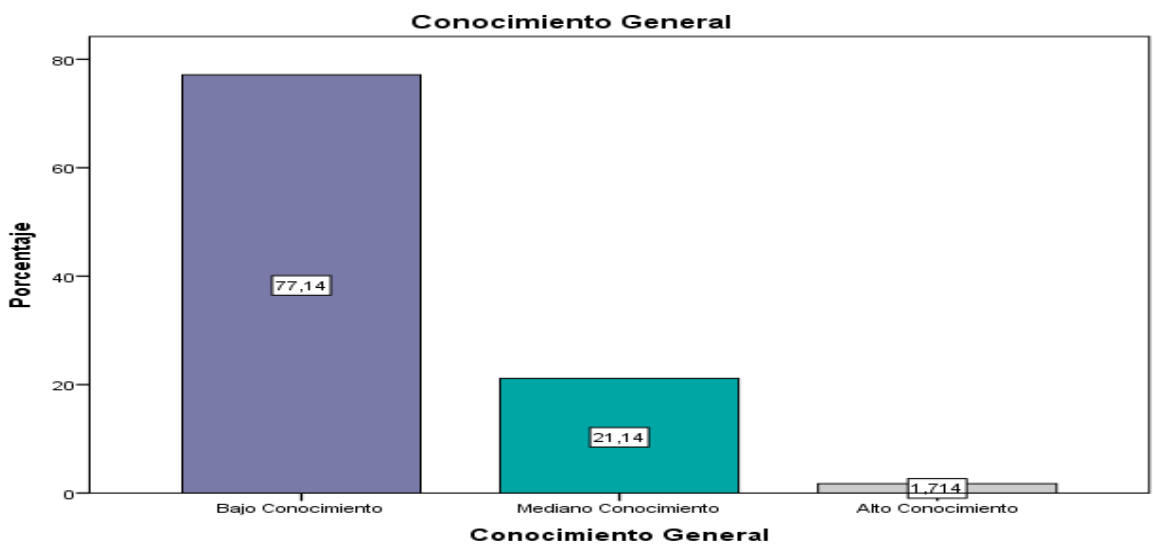


El precio de los productos orgánicos tienden a ser más caros que sus similares convencionales, ello puede ser a causa de menores escalas de producción, controles más estrictos en el proceso productivo, altos costos logísticos entre otros, tal como indican Ramírez (2016) y Amador (2005), por ello, se destaca que más del 50 \% de consumidores concuerdan que los productos orgánicos son más caros o mucho más caros que uno convencional, mientras que al menos el $28 \%$ cree que son más baratos o mucho más baratos, mientras que un $19,43 \%$ indica que los niveles de precio son similares, esta detalle se debiera a que el precio de los productos varía en función de la presentación del producto y el lugar donde se compra.

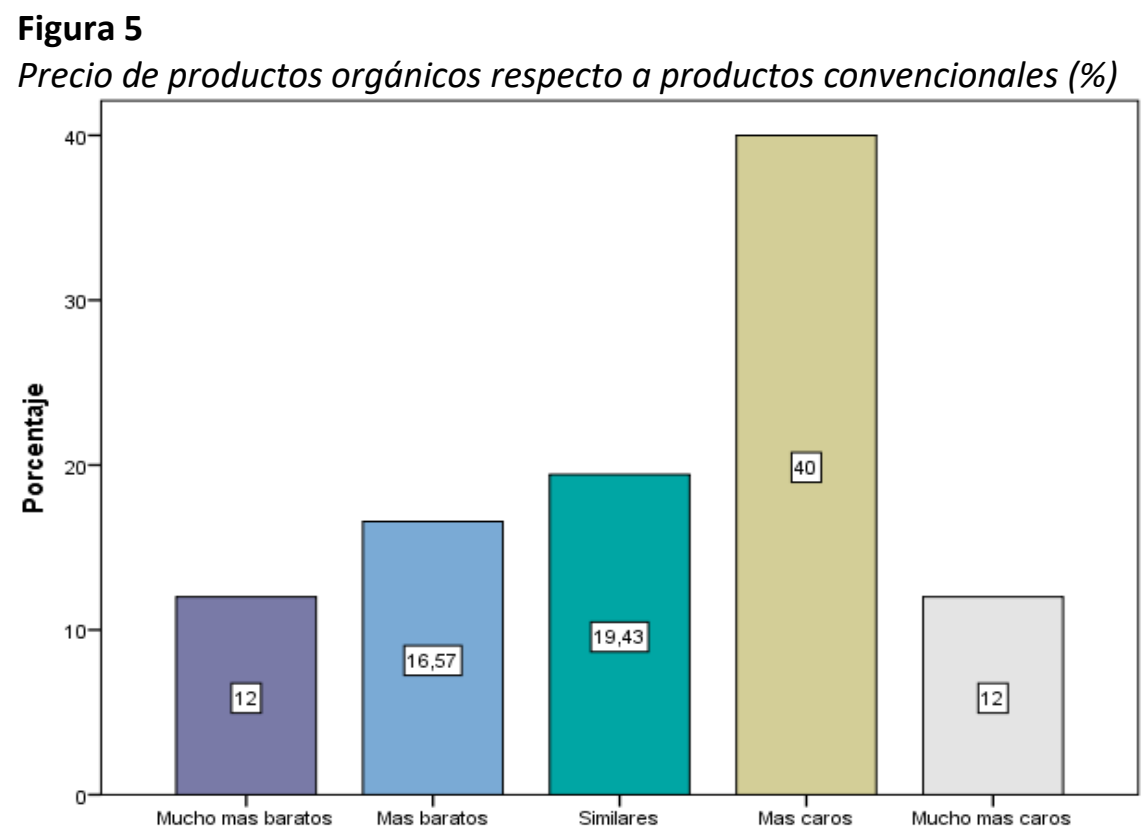

Nota. La representación corresponde a los consumidores declarados

La figura 6, muestra la valoración de sus beneficios, los consumidores manifiestan que consumen productos orgánicos porque son sanos y libres de otras sustancias en aproximadamente 70 $\%$, seguida de un $16,57 \%$ que afirma que son amigables al medio ambiente. Se conoce que en diversos estudios reflejan que los motivos por los que se consume productos orgánicos en función de sus beneficios está principalmente que son saludables, tienen buen sabor o no tienen químicos, así como su capacidad económica para comprarlos (Andrade y Ortiz, 2008; López; 2019; Arellano, 2016 ).

\section{Consumo de productos orgánicos y COVID 19}

Se reportan los resultados de las frecuencias por preguntas efectuadas para esta variable y a partir de ella se agrupó para ver de forma general los efectos que se categorizaron en efectos bajos, efectos medios y efectos altos, a la percepción que tienen los consumidores, si en tiempo de pandemia debido al COVID 19, fue una condicionante para el consumo de productos orgánicos, destacándose que el $46,29 \%$ indica que tuvo un efecto mediano, resultados diferentes se han encontrado en estudios análogos donde el $33 \%$ de la población de Guayaquil indica que aumentó su consumo durante la crisis sanitaria (Macías, 2020). 


\section{Figura 6}

Razones por las que consume productos orgánicos (\%)

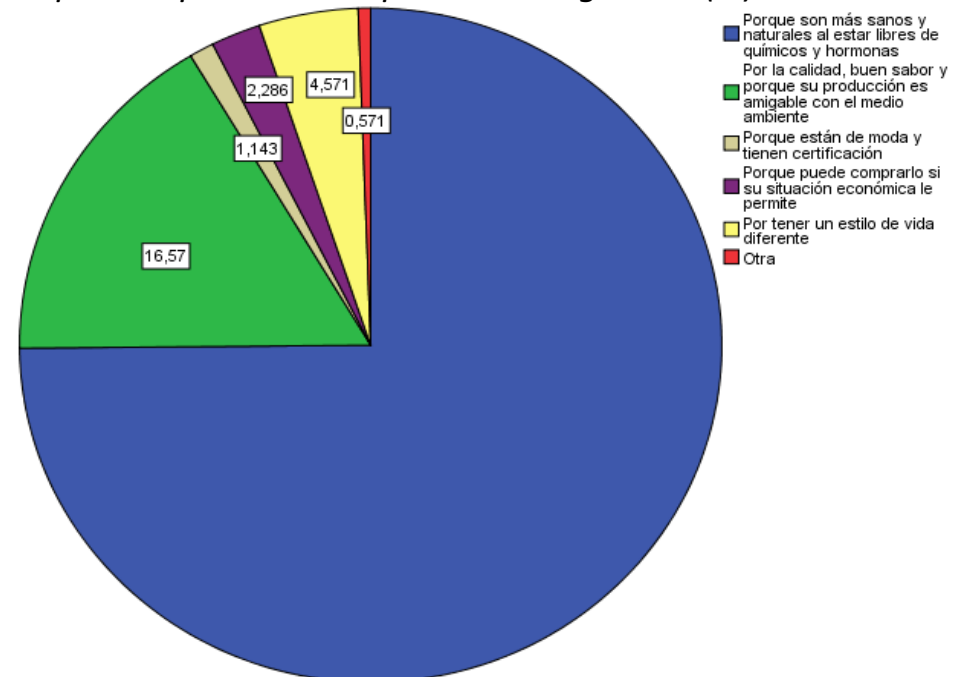

Nota: La representación corresponde a los consumidores declarados

\section{Figura 7}

Efectos del COVID 19 sobre el consumo de productos orgánicos

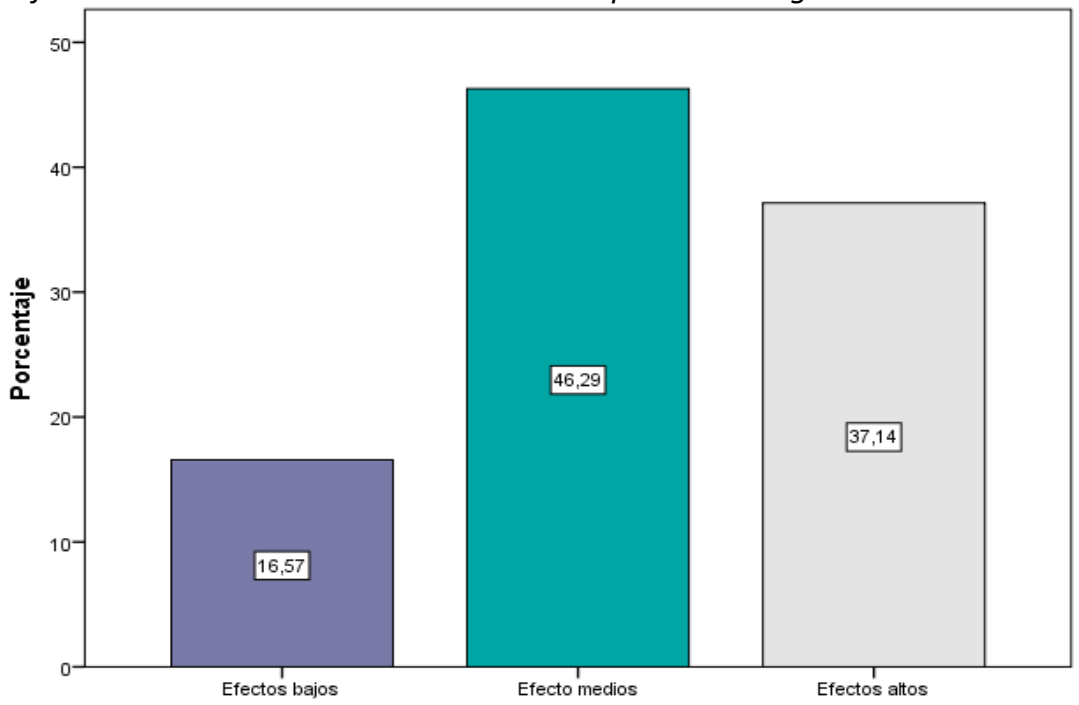

Nota. La representación corresponde a los consumidores declarados

Resultados estadísticos de no consumidores

Las características descriptivas de los que no consumen productos orgánicos ni están interesados en consumirlos se aprecia que se compone del $46,21 \%$ de hombres y $53,8 \%$ de mujeres, las edades están representadas por: el 34,6\% de personas que tienen entre 35 a 45 años; 30,8 \% de personas que tienen entre 46 a 55 .

En cuanto al nivel de estudios destaca el nivel universitario con un $61,5 \%$, seguida de profesionales con grado de maestría o doctorado. El ingreso familiar destaca que el $50 \%$ de no consumidores posee ingresos entre $S / 1300,00$ y S/ 2480,00 . Por otro lado, al explorar el conocimiento que tienen, se encontró que el $30,8 \%$ si conocen y el $69,2 \%$ no tienen conocimiento. 
Al indagar por las razones por los que no consumen, se encuentra diversos motivos tales como: el desconocimiento de sus beneficios (23,1 \%); que son muy caros $(26,9 \%)$; no se encuentran fácilmente $(23,1 \%)$ y tienen duda de que sean orgánicos $(26,9 \%)$. Al hacer inferencias estadísticas con la finalidad de caracterizar las razones por las que no consumen o no están interesados en consumirlos, se realizó una tabla de contingencia y un contraste de Chi cuadrado con el nivel de ingresos, observándose que existe una relación significativa (Sig. $=0,000$ ), entre estas dos variables, es decir existe relación entre las razones de no consumo y el nivel de ingresos, destacándose que mientras menos ingresos tienen, identifican más razones para no consumirlos.

Finalmente, respecto al conocimiento y la variable razón de no consumo se identificó que ambas variables también tienen una relación significativa (Sig. $=0,000$ ), según la tabla de Chi cuadrado es decir entre otras razones, la razón de no consumo se explicaría por el desconocimiento que tienen sobre los productos orgánicos y que mientras a más desconocimiento, mayor posibilidad de no consumo (Rho de Spearman 0,470).

\section{Discusión}

\section{Consumo de Productos Orgánicos}

Existen diversas definiciones de consumidor de productos orgánicos, no obstante, se pueden diferenciar entre los que optan por motivos de salud, otros por afinidad al medio ambiente y otros por moda (López, 2019), estas consideraciones pueden influir en los niveles de consumo, tal como resulta en la presente investigación donde los niveles de consumo son muy bajos, donde solo el 5,7 \% consume a diario, resultados similares se han reportado por Andrade y Florez (2008), donde solo el 5,2\% de población consume con regularidad, niveles más altos que aún son considerados bajos, han sido encontrados por López (2019) quien reporta que solo el 22,7 \% de la población consume y forma parte de su estilo alimenticio. Algunas explicaciones que se pueden esgrimir son las diversas barreras que impiden o hacen difícil el consumo de este tipo de productos; uno de ellos es el alto precio o sobreprecios que presentan a diferencia de un producto convencional, esta afirmación ha sido corroborada en la hipótesis de que los niveles de consumo y precios están relacionados $(\operatorname{Sig}<0,05)$, por lo tanto, el bajo consumo es debido a los precios altos de dichos productos. Otro aspecto que limita el consumo, se debería a la limitada disponibilidad de este tipo de productos ya que en la encuesta se consulta sobre la razón del no consumo de alimentos, donde el 35,3 \% indica que se debe a que este tipo de productos no se encuentran fácilmente por la oferta limitada en la ciudad.

Otra explicación podría ser que el consumo se ve afectado debido a que la mayor cantidad de productos orgánicos son destinados para exportación, finalmente otras que sustentan estas afirmaciones son; que los productos orgánicos no sean realmente orgánicos, lo que genera desconfianza, la corta vida en anaquel en algunos casos; condiciones de vida que no privilegia la salud y el ambiente laboral (López, 2019)

\section{Conocimientos y consumo de productos orgánicos}

Según los resultados obtenidos, los consumidores mostraron bajo conocimiento sobre las propiedades de los productos orgánicos, se destaca de forma general que el $77,14 \%$ de los consumidos tienen un bajo nivel de conocimiento, no obstante la categorización presenta a los consumidores, se puede apreciar que no tendrían un conocimiento pleno de sus propiedades, dichos resultados son similares a los obtenidos por Ortiz (2008) quien encontró que un porcentaje menor (7\%) de población ecuatoriana conoce sobre los productos orgánicos, similares a los obtenidos por Andrade y Florez (2008) quienes determinaron que solo el $3 \%$ de población de la ciudad de Guayaquil conoce los productos orgánicos, en esa dirección los resultados obtenidos por Cruz y Rodríguez (2012) también indican que el $80 \%$ de encuestados no tienen conocimiento sobre los productos orgánicos. Los resultados obtenidos evidenciarían la poca difusión de este tipo de productos y por otro lado la poca 
presencia y penetración de mercado por parte de los productores, sumado a la escasa comercialización y niveles de oferta limitados. Este análisis es confirmado con el contrastes de hipótesis donde se relacionó los niveles de consumo con el conocimiento que mostraron los consumidores, resultando que ambas variables no están relacionadas (sig. $=0,736)$, por lo que se puede afirmar que los niveles de consumo de productos orgánicos no dependen del conocimiento que tienen respecto a ellos, esto es confirmado con el valor de correlación de Spearman $(-0,013)$, que se encontró en un nivel muy bajo y que a la vez muestra que la posible relación es negativa, es decir que a "mayor conocimiento, podría ocasionar menor consumo" esta afirmación no sería coherente con lo teóricamente esperado, donde se espera más bien una correlación.

\section{Nivel de consumo y precios productos orgánicos}

Tal como como lo definen Díaz et al., (2013), el precio es aquel valor monetario otorgado a un producto o servicio según la demanda, en ese sentido los productos orgánicos suelen ser más caros que los convencionales por diversos factores como la poca disponibilidad, controles estrictos en su producción, certificaciones entre otros (Ramírez, 2016; Amador, 2005), al respecto de lo encontrado en la investigación; sugiere que más del $50 \%$ de consumidores perciben que los precios son muy elevados, y la hacer las pruebas de contraste de hipótesis, se verificó que existe una relación significativa entre el nivel de precios y consumo de productos orgánicos (Sig. $=0,05)$, y considerando que las variables son de tipo ordinal, se realizó un análisis no paramétrico mediante la correlación de Spearman cuyo valor es 0,011 indica que es una correlación positiva muy baja, no obstante es importante destacar que al existir correlación positiva, se podría afirmar que al variar una de las variables afectaría directamente a la otra, por lo que el nivel de precios de los productos orgánicos guardan relación al consumo de los mismos, esta posición es compartida por Krystallins y Chyssohoidis (2005) quienes coinciden que al existir una relación positiva, los consumidores están dispuestos a pagar un precio más alto, recalcando que esta asociación es muy débil como se describió anteriormente. También en esa línea es necesario citar a Villaseca (2020), quien, en un estudio sobre precios de productos orgánicos, encontró que los consumidores aseguraron que vale la pena pagar por un precio más alto debido a varios beneficios que provee.

\section{Valoración de beneficios y consumo de productos orgánicos}

La valoración de los beneficios ejerce una mayor influencia en el consumo, principalmente cuando se refiere a salud (Narro y Nayac, 2018), esta acepción es comparativamente igual a lo encontrado en el presente estudio, donde los consumidores dan más peso a los beneficios relacionados a la salud y libres de agroquímicos (aproximadamente el $70 \%$ ) y otro aspecto destacado son los beneficios amigables al medio ambiente $(16,57 \%)$, resultados similares han sido reportados por Cardona (2016), quien encontró que el $83 \%$ de consumidores de Cali en Colombia se encontraban satisfechos con los productos orgánicos identificando entre las principales beneficios asociados a la salud, libres de agroquímicos, contenido nutricional, la calidad y la conservación del medio ambiente. Por otro lado resultados ligeramente inferiores fueron reportados por Palacios y Restrepo (2011) que encontraron que el consumidor de Medellín en un $45 \%$ han consumido alimentos orgánicos, siendo el principal motivo cuidar la salud, también en una investigación realizada por Gómez et al., (2020), refieren que en una escala de 1 a 5 (donde $5=$ total de acuerdo), los consumidores atribuyeron beneficios a la salud $(4,68)$, propiedades nutritivas $(4,67)$ y ausencia de químicos $(4,57)$, no obstante existe una alta valoración en términos de beneficios relacionados a la salud, así como al medio ambiente. Al hacer un análisis de correlación de Chi cuadrado, se encontró que no existe relación entre la valoración de los beneficios y el consumo de productos orgánicos ( $p$-valor $=0,164)$, notándose así mismo que la correlación de Spearman (-0.004), confirma que existe una correlación negativa y muy baja indicando que las variables tienen una relación inversa, la que es teóricamente incorrecto. La explicación estaría en el hecho que, en el estudio se encontró que los consumidores no conocen adecuadamente las propiedades de los productos orgánicos, donde el $77,14 \%$ no tiene un conocimiento adecuado, por lo tanto a pesar de que consumen y valoran beneficios, subyace el factor "desconfianza" por encima de la valoración de beneficios, esta postura fue contrastada con una prueba 
de Chi cuadrado entre las variables valoración de beneficios y la desconfianza de que los productos sean realmente orgánicos, que existe una alta relación significativa ( $P$-valor $=0,000$ ) demostrándose así que la valoración de los beneficios, está más bien condicionada a la desconfianza que existe sobre dichos productos, esta posición es compartida por López (2019) quien indica que en ocasiones hay quienes ponen en duda que los productos orgánicos sean realmente orgánicos.

Se debe tener en cuenta que existen aportes de los productos orgánicos, pero que no están demostrados.

\section{Consumo de productos orgánicos en tiempos de COVID 19}

Se evidencia que los consumidores identificaron que existió efectos tanto medios y altos del COVID 19 sobre el consumo de productos orgánicos, no obstante, estadísticamente no está relacionado a los niveles de consumo, es decir que el consumidor de productos orgánicos concibe que hubo efectos, pero que estos no influyeron sobre sus niveles de consumo.

En esta dimensión, se indagó entre otros; si el consumo de productos orgánicos estaban relacionados con otros factores, por lo que se puede afirmar que los niveles de consumo de productos orgánicos no dependen del conocimiento aumentó durante la pandemia, encontrándose que efectivamente hubo aumento en el consumo (de acuerdo y totalmente de acuerdo $82 \%$ ), dichos resultados son contrarios a los encontrados por Castañeda (2020), quien reportó que el 47,5\% considera que en situación de COVID 19, no se ha dado prioridad al consumo de productos orgánicos, las razones de esta discordancia se basaría en que los consumidores que forman parte del estudio tienen conciencia de tener una alimentación saludable durante la pandemia.

\section{Conclusión}

Se encontró que los niveles de consumo son muy bajos y solo el $5,7 \%$ de los consumidores lo hacen a diario, su explicación estaría en las diferentes barreras como los altos precios, con el cual presentó una relación significativa al $95 \%$ de confianza, por tanto, se concluye que el bajo consumo es debido entre otros factores, a los altos precios en comparación a los productos convencionales.

Los consumidores mostraron bajos niveles de conocimiento respecto a los productos orgánicos, ya que el 77,14 \% desconoce sus propiedades, al relacionar esta variable con los niveles de consumo, se encontró que no existe una relación significativa (sig. $=0.736$ ) por lo tanto los niveles de consumo de productos orgánicos no dependen del conocimiento.

Se encontró que más del $50 \%$ de consumidores percibe que los productos orgánicos tienen precios muy elevados, verificándose que está relacionado directa y significativamente con los bajos niveles de consumo al $95 \%$ de confianza, lo expuesto permite concluir que los niveles altos de precios influyen directamente ocasionando los bajos niveles de consumo.

La valoración de beneficios de los productos orgánicos más destacado por los consumidores está relacionado principalmente a la salud y su contenido libre de agroquímicos en un $70 \%$, no obstante, no se encontró relación significativa de esta variable con los niveles de consumo al $95 \%$ de confianza. Finalmente, se encontró que la pandemia COVID 19, sí tuvo efectos sobre el consumo, determinándose que, si bien existieron efectos tanto medios y altos, estadísticamente no se evidenció relación significativa con la variación de los niveles de consumo al $95 \%$ de confianza. 


\section{Referencias Bibliográficas}

Amador, L. (2005). Productos orgánicos retorno a lo natural. PROFECO. 58-62. Recuperado de https:// www.profeco.gob.mx/revista/publicaciones/adelantos_05/organicos_oct05.pdf

Andrade, C. M., y Ayaviri, D. (2018). Demanda y consumo de productos orgánicos en el cantón Riobamba, Ecuador. Información tecnológica, 29(4), 217-226.

Bermejo Mallea, G. F. (2018). Factores que Influyen en la Intención de Compra de Alimentos Orgánicos, en los Consumidores del Distrito Coronel Gregorio Albarracín Lanchipa, 2018.

Cardona, M. M. (2016). Alimentos orgánicos: factores de satisfacción del consumidor. Caderno Profissional de Marketing-UNIMEP, 4(1), 58-68. Recuperado de https://www.cadernomarketingunimep.com.br/ojs/index.php/cadprofmkt/article/view/48

Cardozo Palomares, A. F., Chavez Carrillo, R. L., Porras Aldavarca, J. G., \& Tarazona Suarez, E. E. (2019). Plan de negocio sobre delivery de comida saludable a base de insumos orgánicos: Organik.

Castañeda Suarez, S. (2020). Bajo consumos de productos Orgánicos en Itagüí. Disponible en https://n9.cl/f8al7

Cisneros G. (2012). Salud en un bocado. En: Revista Dionisos, año 14, № 89, pp. 80.

Cruz Roa, G. S., \& Rodriguez Velasco, P. A. (2012). Sistema de información para la comercialización y gestión del conocimiento en comunidades de interés de productos orgánicos. Disponible en https://n9.cl/t2m5

Díaz, I., Cataluña, F., Díez de Castro, E. (2013) Gestión de precios. Recuperado de https://n9.cl/s5gt

Gentile, N., \& Rodríguez, E. M. M. (2002). El consumo de alimentos orgánicos: Aplicación de un modelo logit multinomial en la elección del canal de compra. In XXXVII Reunión Anual de la Asociación Argentina de Economía Política.

Gob.Pe (2021). Productores de la Región Tacna concretan venta de orégano orgánico con asistencia técnica de Sierra y Selva Exportadora. (2020). [Comunicado de prensa] Recuperado de website: https://n9.cl/c59r

Gómez, B. O. C., Valenzuela, D. M. R., y Gómez, D. M. C. (2020). Alimentos orgánicos en Hermosillo, Sonora: disposición a pagar y preferencias del consumidor. Estudios Sociales: Revista de Alimentación Contemporánea y Desarrollo Regional, 30(55), 1.

Hernández, R., Fernández, C. y Baptista, Pilar. (2010). Metodología de la investigación 5a ed.). México: McGraw-Hill.

Higuchi, A. (2015). Características de los consumidores de productos orgánicos y expansión de su oferta en Lima. Apuntes, 42(77), 57-89.

Ipsos. 2021. Características de los niveles socioeconómicos en el Perú. [Online] Available at: <https://www.ipsos.com/es-pe/caracteristicas-de-los-niveles-socioeconomicos-en-el-peru> [Accessed 19 February 2021].

Jurado, C. (2009) Consumidores ecológicos en acción. En: Leisa. Revista de Agroecología, vol. 25, $\mathrm{N}^{\circ} 4$, pp. 35-36. Disponible en https://n9.cl/2sum

Krystallins, A., y Chryssohoidis, G. (2005) Consumers' willingness to pay for organic food: Factors that affect it and variation per organic product type. Recuperado de http://doi.org/10.1108/00070700510596901

Lacaze, V. (2009). Consumos alimentarios sustentables en Argentina: una estimación de la disposición a pagar por alimentos orgánicos frescos y procesados por consumidores de la ciudad de Buenos Aires. Agroalimentaria, 15(29), 87-100.

Lipovetsky, G., \& Naranjo, L. G. G. (1999). La tercera mujer. Barcelona: Anagrama.

Lutterbeck, B. (2001). Más Sano Y Con Sabor Más Natural: Quiénes Compran Alimentos Orgánicos Y Por Qué. Comuniica Magazine, 2001(936-2016-74367), 23-25.

Narro Ríos, R. M., y Yánac Gaitán, R. H. (2018). Influencia del nivel de conocimiento y la valoración de beneficios de los alimentos orgánicos en los consumidores de los NSE A y B en Lima 
Metropolitana. Universidad Peruana de Ciencias Aplicadas (UPC), Lima, Perú. Doi: https://doi.org/10.19083/tesis/624573

Ortega Bailón, G. F. (2013). Factores que influyen en la adopción de tecnologías orgánicas por los productores olivareros de La Yarada.

Ortiz, D. A., \& Flores, M. (2008). Consumo de productos orgánicos/agroecológicos en los hogares ecuatorianos. VECO, Quito, Ecuador. Disponible en https://n9.cl/md4v

Palacios, M., y Restrepo, A. (2011). Caracterización conductual de los consumidores de restaurantes orgánicos de los estratos 4, 5 y 6 de Medellín. (Tesis de pregrado). Escuela de Ingeniería de Antioquia, Facultad de Ingeniería Administrativa, Envigado, Colombia.

Paul, J., \& Rana, J. (2012). Consumer behavior and purchase intention for organic food. Journal of consumer Marketing, 29(6), 412-422

Posada, C. (2018). Productos orgánicos cobran mayor interés en mercados internacionales. La Cámara, 26. Disponible en https://n9.cl/vkr46

Ramírez, M. (2016). El mercado de productos orgánicos crece $10 \%$ cada año. Milenio. Recuperado http:// comer sano 0 708529215.html

Republica. (3 de marzo de 2021). ¿Por qué no se consumen más alimentos orgánicos? Google. Republica Recuperado de https://gastronomiaycia.republica.com/2011/02/17/por-que-no-seconsumen-mas-alimentos-organicos/

Reyes, J., Rubina, M., \& Sierra, J. (2014). Plan de marketing para el lanzamiento de un minimarket de productos orgánicos (Doctoral dissertation, Tesis de Magister en Dirección de Marketing y Gestión Comercial, Universidad Peruana de Ciencias Aplicadas. Recuperado de http://repositorioacademico. Upc. Edu. Pe/upc/handle/10757/62089).

Ríos-González, C. M., y De Benedictis, G. A. (2017). Predisposición para la participación de estudiantes de medicina en encuestas online: una reflexión sobre la experiencia. FEM: Revista de la Fundación Educación Médica, 20(4), 205-205.

Salgado-Beltrán, L., y Beltrán-Morales, L. F. (2011). Factores que influyen en el consumo sustentable de productos orgánicos en el noroeste de México. Universidad y ciencia, 27(3), 265-279.

Saucedo. (2010). Características, limitaciones y posibilidades de desarrollo de la producción y comercialización de productos orgánico en el Perú. Recuperada de: http://repositorio.lamolina.edu.pe

Servicio Nacional de producción agraria - SENASA (2018). Estadísticas De Producción Orgánica

https://www.senasa.gob.pe/senasa/descargasarchivos/2019/07/Cuadro-1-2018ESTAD\%C3\%8DSTICAS-DE-PRODUCCI\%C3\%93N-ORG\%C3\%81NICA-NACIONAL-.pdf

Soto, B. (2015). Página web de Actualidad Ambiental. Disponible en https://www.actualidadambiental.pe/category/general/

Villaseca Aparicio, V. (2020) Estrategia de precio elevado en productos orgánicos y la calidad percibida en relación con la intención de compra en tiendas orgánicas de Lima. Disponible en https://n9.cl/xfewq 